Review began 02/20/2022 Review ended 02/22/2022 Published 02/23/2022

๑) Copyright 2022

Rugbeer et al. This is an open access article distributed under the terms of the Creative Commons Attribution License CCBY 4.0., which permits unrestricted use, distribution, and reproduction in any medium, provided the original author and source are credited.

\section{The Ace of Spades: Apical Hypertrophic Cardiomyopathy in an African Male Patient}

\author{
Yashvir Rugbeer ${ }^{1}$, Muhammad Ismail ${ }^{1}$, Sandhiran Nadar ${ }^{2}$, Darrin R. Naidoo ${ }^{2}$ \\ 1. Department of Internal Medicine, Grey's Hospital, Pietermaritzburg, ZAF 2. Department of Cardiology, Inkosi Albert \\ Luthuli Central Hospital, Durban, ZAF
}

Corresponding author: Yashvir Rugbeer, yashvir77@gmail.com

\begin{abstract}
We present a case of a 52-year-old African male patient diagnosed with apical hypertrophic cardiomyopathy. He was initially diagnosed with hypertensive heart disease and placed on anti-failure treatment. Following multiple subsequent presentations and on closer review of his signs and symptoms, apical hypertrophic cardiomyopathy was considered. The diagnosis was made five years after his initial presentation and confirmed by echocardiography and cardiac magnetic resonance imaging. This case report explores his presentation and aims to provide further literature on the aetiology, description, and management of apical hypertrophic cardiomyopathy, particularly within the African population.
\end{abstract}

Categories: Cardiology, Internal Medicine

Keywords: magnetic resonance imaging, electrocardiogram, echocardiography, left ventricular hypertrophy, apical hypertrophic cardiomyopathy

\section{Introduction}

Hypertrophic cardiomyopathy (HCM) describes myocardial disease with left ventricular hypertrophy (LVH) in the absence of a precipitating cardiac or systemic cause [1]. HCM is generally well described, and although likely to be evenly distributed among different populations worldwide, it is noted to be especially rare in the African setting [1,2]. Further literature of its incidence, prevalence and presentation within the African population is required, which will ultimately guide effective management and prevention strategies [2]. Apical hypertrophic cardiomyopathy (Ap HCM) is a rare variant of HCM, and was first described in a case report published in Japan in 1976 [1,3,4,5]. In the largest study of a cohort of patients with HCM who had undergone investigation, a $<10 \%$ prevalence of Ap HCM was noted [1,6,7,8]. The mean age of presentation of Ap HCM is $41 \pm 14.5$ years, and has a male to female ratio of 1:5 [9].

In a retrospective study of 105 patients diagnosed with Ap HCM conducted in Toronto in 2002, 56\% of patients were symptomatic upon diagnosis and follow-up [10]. The commonest symptom experienced was exertional dyspnoea (38\%), followed by palpitations (21\%), typical angina (18\%), atypical chest pain (14\%), pre-syncopal episodes (10\%) and syncopal episodes (6\%) [10]. Examination findings include a sustained, forceful double apical impulse, which is laterally displaced [11]. Auscultation may reveal a normal first heart sound (S1), a split second heart sound (S2), and an audible fourth heart sound (S4), with or without a crescendo-decrescendo, mid-diastolic murmur heard loudest at the lower left sternal border $[4,9,11]$. Decompensated left ventricular function and subsequent pulmonary hypertension can present with the classic clinical signs and symptoms of bi-ventricular failure, such as exertional dyspnoea, orthopnoea, paroxysmal nocturnal dyspnoea, a raised jugular venous pulsation, and the presence of a third heart sound (S3) [12]. The complications of Ap HCM, as noted in the aforementioned retrospective Toronto study in 2002, included atrial fibrillation (12\%), myocardial infarction (10.5\%), congestive cardiac failure (5\%), and ventricular fibrillation (1\%) [10]. Overall mortality was noted to be $10.5 \%$, with a cardiovascular mortality of $1.9 \%$ and an annual cardiovascular mortality of $0.1 \%$ [10]. Diagnostic criteria include asymmetrical left ventricular hypertrophy mostly confined to the apex of the heart, an apical wall thickness of $\geqslant 15 \mathrm{~mm}$, and a ratio of maximal apical to posterior wall thickness of $\geqslant 1.5 \mathrm{~mm}$ based on echocardiography (ECHO) or cardiac magnetic resonance imaging (MRI) $[1,4,6,9]$. Typical electrocardiography (ECG) findings are negative Twaves in the precordial leads with a depth of $\geqslant 10 \mathrm{~mm}$, and evidence of left ventricular hypertrophy $[3,9]$. Cardiac ventriculography may demonstrate the typical “ace-of-spades"-like configuration in the left ventricle, along with marked apical obliteration [1]

\section{Case Presentation}

A 52-year-old African male patient with no known medical co-morbidities or prior cardiac history presented to the emergency department of a tertiary level hospital in September 2016, having been referred from his base hospital for further investigation and management. His symptoms included a one-day history of progressively worsening typical cardiac chest pain (retrosternal chest discomfort worsened by exertion and relieved by rest [13] ), nausea, and diaphoresis. Table 1 summarizes his relevant clinical findings on the initial presentation. 


\section{Cureus}

Category Clinical Findings

Vital signs Pulse: 60 beats per minute, regular rhythm and normal volume, blood pressure: $146 / 92 \mathrm{mmHg}$, respiratory rate: 18 breaths per minute, temperature: $36.8^{\circ} \mathrm{C}$, oxygen saturation: $98 \%$ in ambient room air, blood glucose: $5.8 \mathrm{mmol} / \mathrm{L}$

General Comfortable at rest. Absence of clubbing, pallor, and pedal oedema. No signs of central or peripheral cyanosis, plethora or examination diaphoresis.

Cardiac No signs of infective endocarditis. A non-elevated, normal morphology jugular venous pulsation. Normal precordium, no system examination parasternal heave, thrills, or palpable pulmonary component of the second heart sound (P2). Displaced apex beat to the left $6^{\text {th }}$ intercostal space in the anterior axillary line. Normal first heart sound (S1) with a loud aortic component of the second heart sound (A2). No additional heart sounds/murmurs.

Respiratory

system Chest fields clear with good air entry bilaterally. No crepitations or features of costochondritis.

examination

Abdominal

system Soft and non-tender. No organomegaly.

examination

Nervous

system Oriented to time, person and place. No focal neurological signs.

examination

TABLE 1: Clinical findings on presentation

Laboratory investigations were normal, apart from an elevated cardiac troponin-I level of $1185 \mathrm{ng} / \mathrm{L}$ taken 16 hours following the onset of chest pain, and an elevated creatine kinase (CK) level of $268 \mathrm{U} / \mathrm{L}$. The creatine kinase MB (CK-MB) mass was of a normal value of $2.70 \mathrm{ug} / \mathrm{L}$, and a repeat troponin-I level taken six hours later was of a value of $1122 \mathrm{ng} / \mathrm{L}$ (a $5 \%$ decrement from the initial value). Figure 1 illustrates the electrocardiogram (ECG) taken on initial presentation and describes its findings. Echocardiography noted an end-diastolic left ventricular internal dimension measured at $4.9 \mathrm{~cm}$ (normal range: $4.2 \mathrm{~cm}-5.8 \mathrm{~cm}$ ), an ejection fraction of $65 \%$, and no valvular or pericardial abnormalities. Cardiac angiography noted normal epicardial arteries with a hocoid left ventricle; left ventriculography showed a typical "ace-of-spades"-like configuration of the left ventricular cavity (See Figure 2).

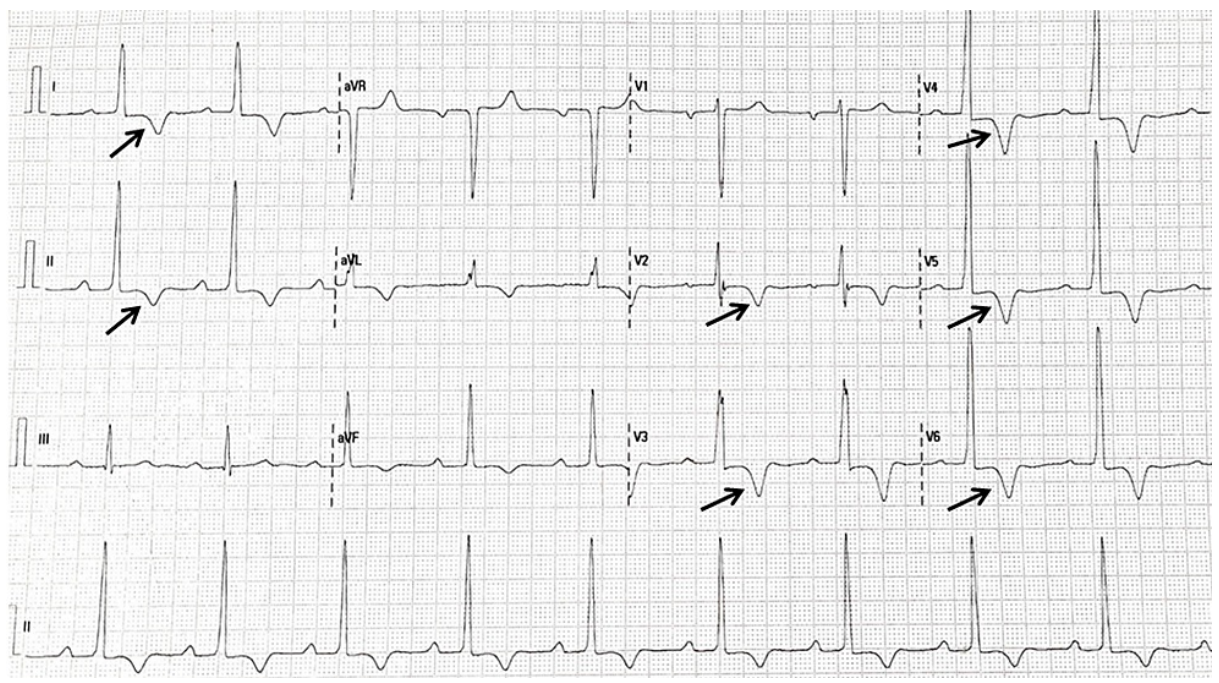

\section{FIGURE 1: ECG of the patient on initial presentation}

A sinus rhythm with a rate of 60 beats per minute and a normal electrical axis is noted, along with left ventricular hypertrophy according to voltage criteria and $1 \mathrm{~mm} \mathrm{ST-segment} \mathrm{depression.} \mathrm{Arrows} \mathrm{illustrate} \mathrm{inverted} \mathrm{T-waves} \mathrm{of} \mathrm{>}$ $5 \mathrm{~mm}$ in Leads I, II, and V2-V6. 


\section{Cureus}

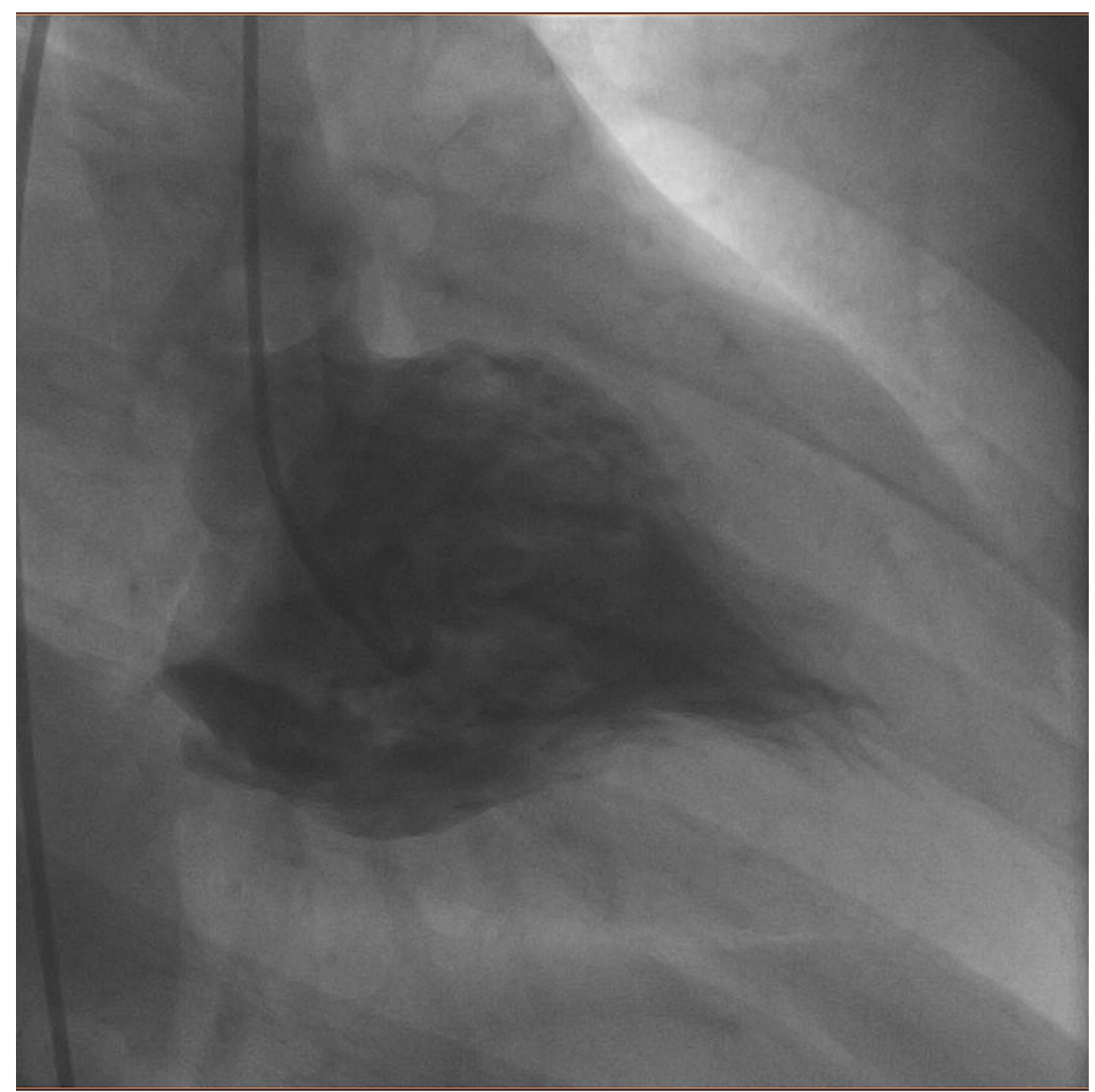

\section{FIGURE 2: Left ventriculography performed on the patient}

The image illustrates a typical "ace-of-spades"-like configuration of the left ventricle.

An initial diagnosis of hypertensive heart disease with elevated serum troponin levels was made. The patient was discharged and placed on enalapril $2.5 \mathrm{mg}$ bi-daily, aspirin $150 \mathrm{mg}$ daily, and atorvastatin $40 \mathrm{mg}$ daily. $\mathrm{He}$ presented a further two times with similar presentations but was again discharged with the above medication. In September 2020, he once again presented to the emergency unit, now complaining of presyncopal symptoms and multiple syncopal episodes. On close review of his investigative profile along with repeat ECG and echocardiography, a diagnosis of Ap HCM was considered. Cardiac MRI was performed, which noted significant myocardial wall thickening of $23 \mathrm{~mm}$ (See Figure 3), and near-complete cavity obliteration of the apex (See Figure 4 and Figure 5). Features were in keeping with predominant apical hypertrophic obstructive cardiomyopathy, along with myocardial infiltration and fibrosis (See Figure 6). A definitive diagnosis of Ap HCM was reached, and the patient was placed on Atenolol 25mg daily and Amiodarone 400mg daily. He unfortunately demised six months later, with sudden cardiac death being the presumed cause. 


\section{Cureus}

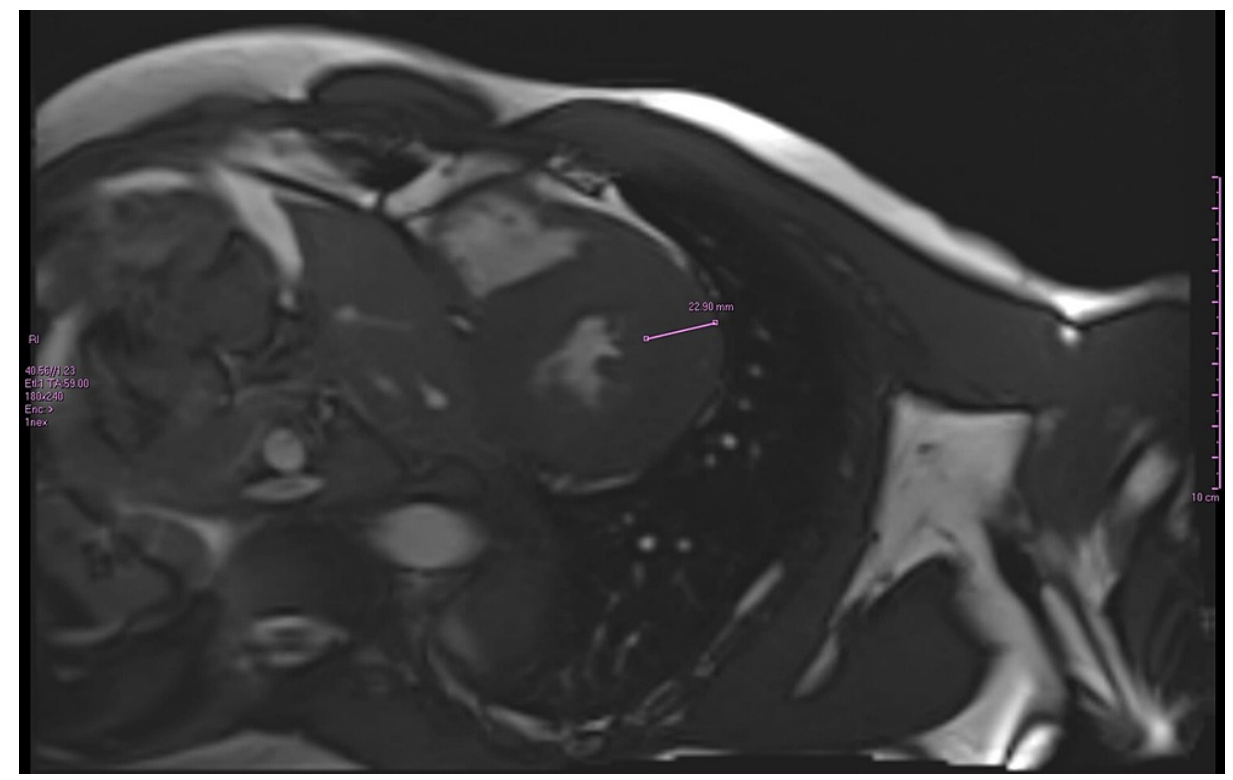

FIGURE 3: Cardiac MRI of the patient

Cine image of the short oblique-axis of the left ventricle. The thickness of the basal anterior lateral wall (purple arrow span) is measured at $23 \mathrm{~mm}$ (normal values of $6.5 \mathrm{~mm}-8.5 \mathrm{~mm}$ ), demonstrating significant myocardial thickening. 


\section{Cureus}

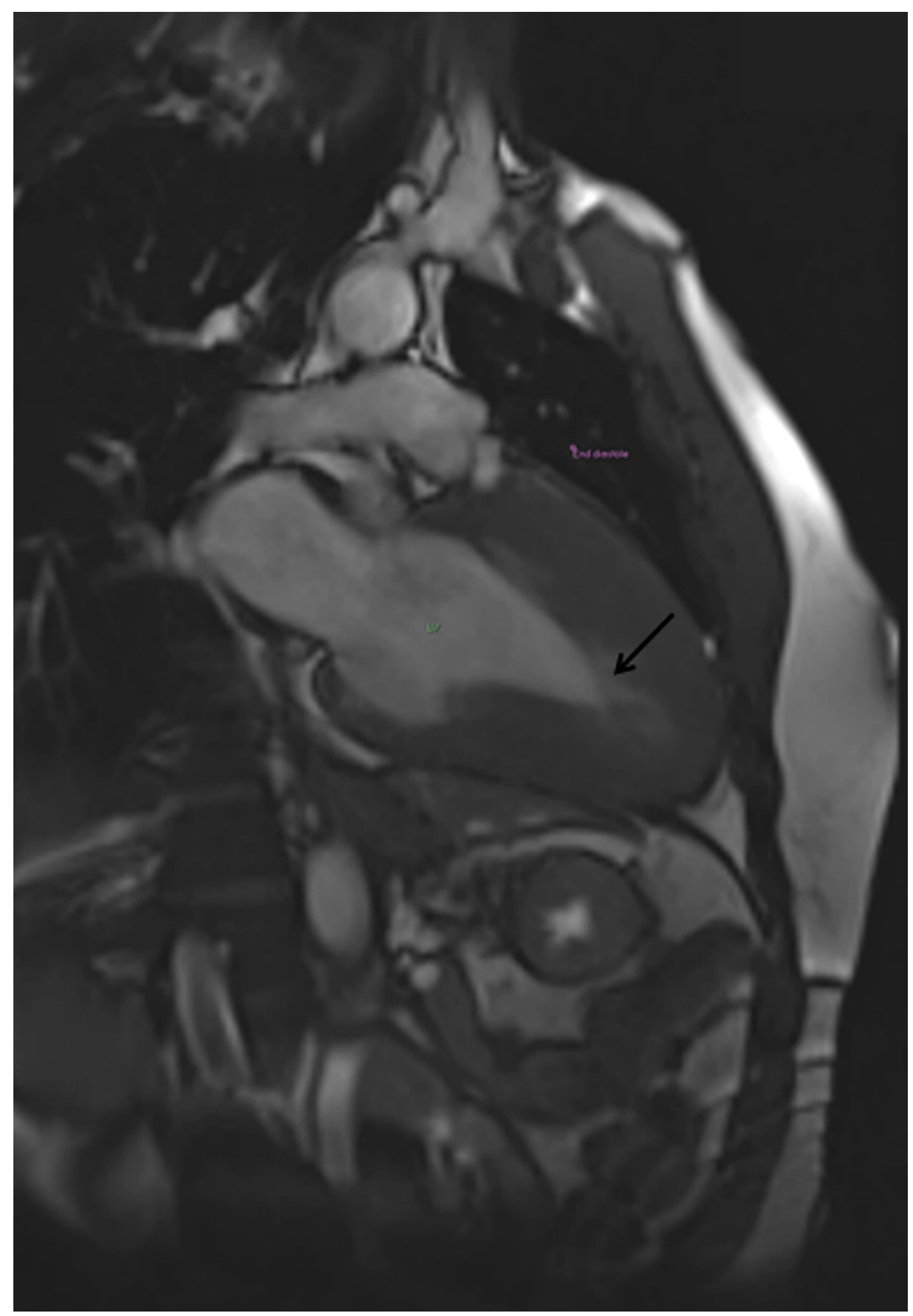

FIGURE 4: Two-chamber view (end-diastole)

Arrow indicates near-complete cavity obliteration of the apex in end-diastole. 


\section{Cureus}

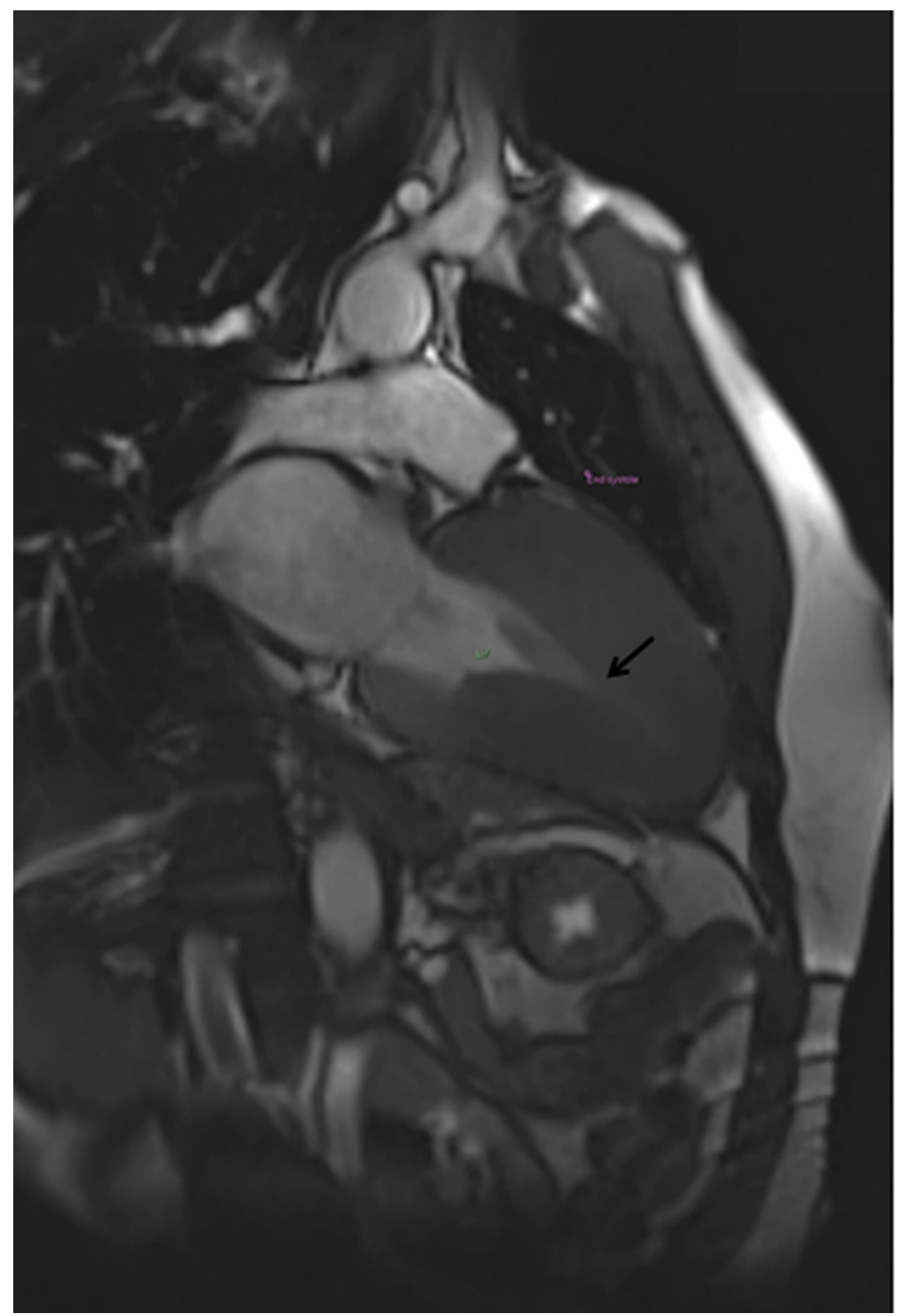

FIGURE 5: Two-chamber view (end-systole)

Arrow indicates near-complete cavity obliteration of the apex in end-systole. 


\section{Cureus}

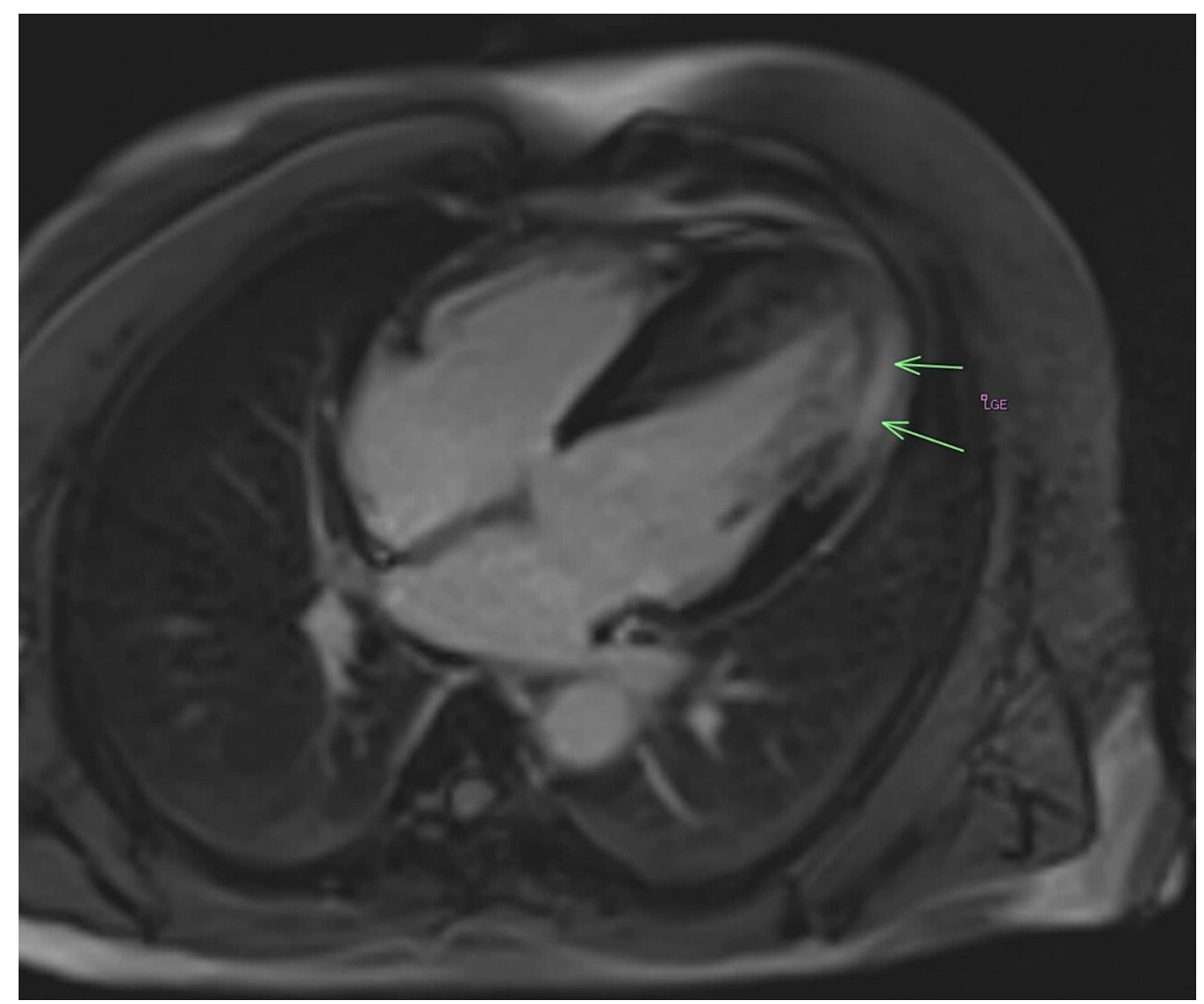

\section{FIGURE 6: Four-chamber view with gadolinium enhancement}

Arrows indicate late gadolinium enhancement: Significant mid-wall myocardial enhancement of the mid-ventricle and apical lateral wall is observed, indicating significant fibrosis.

\section{Discussion}

Multiple different morphologically distinct patterns of hypertrophic cardiomyopathy are described [1]. The commonest sub-types are asymmetrical septa, concentric, reverse septal, neutral, and apical, along with other rarer forms [1]. It is further sub-divided into three types, being either pure focal, pure diffuse, or a combination of the two [1]. The aetiology of Ap HCM is hereditary, with an autosomal dominant mode of inheritance [7,14]. The condition results from mutations in sarcomeric protein genes; however, these mutations were found in less than a quarter of patients diagnosed with the disease who had undergone genetic studies [7]. The commonest genotypes were noted to be mutations in the myosin-binding protein C (MYBPC3) gene, and the myosin heavy-chain beta 7 (MYH7) gene located on chromosome 11q11 [7,14]. The diagnostic approach involves a comprehensive assessment of the patient, including close attention to symptoms and clinical examination. Investigations such as ECG, ECHO, and MRI may confirm the diagnosis, with risk stratification via the American College of Cardiology/European Society of Cardiology (ACC/ESC) algorithm also necessary $[1,9]$. Management options are categorized into optimal medical treatment, surgery, and ablation, as well as specialized cardiac devices such as implantable cardioverter-defibrillators [9]. Medications such as beta-blockers, calcium channel blockers, and antiarrhythmic agents are commonly used in symptomatic patients, with the objectives of alleviating symptoms as well as decreasing the incidence of events such as arrhythmias and sudden cardiac death [9]. Angiotensin-converting-enzyme (ACE) inhibitors, angiotensin receptor blockers (ARBs), and aldosterone receptor antagonists are not recommended, as their action may exacerbate symptoms and further predispose to pre-syncopal/syncopal episodes by further reducing afterload and increasing outflow gradient [15]

The diagnosis of Ap HCM was only considered five years following our patient's initial presentation. This could be due to the relative rarity of the disease in the African population combined with the challenge of severely resource-limited settings. The hospital where the patient was eventually diagnosed and managed provides a tertiary level healthcare service (i.e., specialised health care) to approximately 3.5 million patients across five different health districts. This results in extremely limited access to investigative resources (such as ECHO and MRI), as well as specialist cardiology consultation. Unfortunately, the delay in diagnosis along with constrained management options subsequently contributed to the poor outcome seen in our patient. The lack of availability of genetic testing for the condition in our setting also results in difficulty in excluding the disease in first degree relatives, where early diagnosis would prevent long-term morbidity and mortality. 


\section{Conclusions}

Ap HCM introduces an interesting caveat to the spectrum of HCM, and is known to be of very low incidence in the African population. Diagnosis requires a high index of suspicion and close attention to a patient's profile, with early surveillance and management necessary to reduce long-term morbidity and mortality. Due to its genetic basis, this not only holds true for the affected patient, but also for first degree relatives. We suggest that Ap HCM be more frequently considered with suggestive patient presentation and examination, along within the disease spectrum of HCM. Further research and literature of the condition, especially within the African population, would prove to be of value.

\section{Additional Information \\ Disclosures}

Human subjects: Consent was obtained or waived by all participants in this study. Conflicts of interest: In compliance with the ICMJE uniform disclosure form, all authors declare the following: Payment/services info: All authors have declared that no financial support was received from any organization for the submitted work. Financial relationships: All authors have declared that they have no financial relationships at present or within the previous three years with any organizations that might have an interest in the submitted work. Other relationships: All authors have declared that there are no other relationships or activities that could appear to have influenced the submitted work.

\section{Acknowledgements}

The authors would like to thank Dr. Miranda Durand, Bachelor of Medicine, Bachelor of Surgery (MbChb) University of Kwazulu-Natal (UKZN), Fellowship of the College of Diagnostic Radiologists of South Africa (FC Rad Diag SA), Department of Radiology, Grey's Hospital, UKZN, for her invaluable radiological contributions.

\section{References}

1. Hughes RK, Knott KD, Malcolmson J, et al.: Apical hypertrophic cardiomyopathy: the variant less known. J Am Heart Assoc. 2020, 9:e015294. 10.1161/JAHA.119.015294

2. Sliwa K, Damasceno A, Mayosi BM: Epidemiology and etiology of cardiomyopathy in Africa . Circulation. 2005, 112:3577-83. 10.1161/CIRCULATIONAHA.105.542894

3. Ho AM, Chui PT, Lee AP, Wan S: Apical hypertrophic cardiomyopathy. Anesth Analg. 2015, 121:1398-9. 10.1213/ANE.0000000000000867

4. Mirabbasi SA, Khalighi K, Mukkamala S, Kodali A: A rare case of apical hypertrophic cardiomyopathy (AHCM). J Community Hosp Intern Med Perspect. 2017, 7:122-5. 10.1080/20009666.2017.1324238

5. Paluszkiewicz J, Krasinska B, Milting H, Gummert J, Pyda M: Apical hypertrophic cardiomyopathy: diagnosis, medical and surgical treatment. Kardiochir Torakochirurgia Pol. 2018, 15:246-53. 10.5114/kitp.2018.80922

6. O'Mahony C, Jichi F, Ommen SR, et al.: International external validation study of the 2014 European Society of Cardiology guidelines on sudden cardiac death prevention in hypertrophic cardiomyopathy (EVIDENCEHCM). Circulation. 2018, 137:1015-23. 10.1161/CIRCULATIONAHA.117.030437

7. Towe EC, Bos JM, Ommen SR, Gersh BJ, Ackerman MJ: Genotype-phenotype correlations in apical variant hypertrophic cardiomyopathy. Congenit Heart Dis. 2015, 10:E139-45. 10.1111/chd.12242

8. Files Flores R, Mané F, Antunes N, Pereira VH: Apical hypertrophic cardiomyopathy, are low-risk patients really at low risk? A case report. Eur Heart J Case Rep. 2020, 4:1-4. 10.1093/ehjcr/ytaa316

9. Yusuf SW, Bathina JD, Banchs J, Mouhayar EN, Daher IN: Apical hypertrophic cardiomyopathy. World J Cardiol. 2011, 3:256-9. 10.4330/wjc.v3.i7.256

10. Eriksson MJ, Sonnenberg B, Woo A, Rakowski P, Parker TG, Wigle ED, Rakowski H: Long-term outcome in patients with apical hypertrophic cardiomyopathy. J Am Coll Cardiol. 2002, 20:638-45. 10.1016/s07351097(01)01778-8

11. Basit H, Brito D, Sharma S: Hypertrophic Cardiomyopathy. StatPearls [Internet]. StatPearls Publishing LLC, Treasure Island, FL; 2022. https://pubmed.ncbi.nlm.nih.gov/28613539/:

12. Malik A, Brito D, Vaqar S, Chhabra L: Congestive heart failure. StatPearls [Internet]. StatPearls Publishing, Treasure Island, FL; 2021.

13. Talley NJ, O'Connor S: The cardiovascular history. A Systematic Guide to Physical Diagnosis, Seventh Edition. Elsevier, Churchill Livingstone, Sydney, Australia; 2014. 45-55.

14. Marian AJ, Roberts R: The molecular genetic basis for hypertrophic cardiomyopathy. J Mol Cell Cardiol. 2001, 33:655-70. 10.1006/jmcc.2001.1340

15. Houston BA, Stevens GR: Hypertrophic cardiomyopathy: a review. Clin Med Insights Cardiol. 2014, 8:53-65. 10.4137/CMC.S15717 